\title{
REPRESENTATIONS AND $K$-THEORY OF DISCRETE GROUPS
}

\author{
ALEJANDRO ADEM
}

\begin{abstract}
Let $\Gamma$ be a discrete group of finite virtual cohomological dimension with certain finiteness conditions of the type satisfied by arithmetic groups. We define a representation ring for $\Gamma$, determined on its elements of finite order, which is of finite type. Then we determine the contribution of this ring to the topological $K$-theory $K^{*}(B \Gamma)$, obtaining an exact formula for the difference in terms of the cohomology of the centralizers of elements of finite order in $\Gamma$.
\end{abstract}

\section{INTRODUCTION}

Let $\Gamma$ denote a discrete group of finite virtual cohomological dimension. Examples of this type of group include finite groups, arithmetic groups, and mapping class groups, making them an important class of objects in both topology and algebra. In particular, understanding the classifying space $B \Gamma$ for such groups is a central problem in algebraic topology. Unfortunately, the cohomology $H^{*}(B \Gamma, \mathbb{Z})$ is a very intractable object; consequently, there are few available calculations (e.g., see [So]). In sufficiently high dimensions the cohomology is known to depend only on the lattice $\mathscr{F}$ of finite subgroups in $\Gamma$ $[\mathrm{B}, \mathrm{F}]$, but in general this yields a complicated spectral sequence involving the cohomology of the normalizers $N(S), S \in \mathscr{F}$.

In this note we outline an approach to understanding the rôle of representations in the topology of $B \Gamma$ as was done in the case of finite groups by Atiyah [At]. We define a representation ring determined on the elements of finite order in $\Gamma$, which for a large class of groups (including arithmetic groups) is of finite rank. Then we indicate to what degree the topological $K$-theory $K^{*}(B \Gamma)$ is determined by these representations. In fact, we provide a precise description of the discrepancy in terms of the rational cohomology of the centralizers of elements of finite order in $\Gamma$. Complete details will appear elsewhere.

\section{A REDUCED REPRESENTATION RING FOR $\Gamma$}

From now on we will assume that $\Gamma$ has a finite number of distinct conjugacy classes of elements of finite order and that their centralizers are homologically finite. These hypotheses are known to hold, in particular, for arithmetic groups.

Definition 1.1. Let $V, W$ be two finite-dimensional $\mathbb{C} \Gamma$-modules. We say that $V$ is $\mathscr{F}$-isomorphic to $W$ if $\left.\left.V\right|_{S} \cong W\right|_{S}$ for all $S \in \mathscr{F}$.

Definition 1.2. $R_{\mathscr{F}}(\Gamma)$ is the Grothendieck group on $\mathscr{F}$-isomorphism classes of finite-dimensional $\mathbb{C} \Gamma$-modules.

Received by the editors May 20, 1992.

1991 Mathematics Subject Classification. Primary 55R35.

Research partially supported by an NSF grant. 
We can, of course, also describe $R_{\mathscr{F}}(\Gamma)$ as a quotient of the usual representation ring $R(\Gamma)$. Let $n(\Gamma)$ denote the number of distinct conjugacy classes of elements of finite order in $\Gamma$. Using character theory arguments, we prove

Proposition 1.3. $R_{\mathscr{F}}(\Gamma)$ is a commutative, unitary ring, which as an abelian group is free of rank $n(\Gamma)$. In particular, $\Gamma$ is torsion-free if and only if $R_{\mathscr{F}}(\Gamma) \cong$ $\mathbb{Z}$.

Similarly, if $\mathscr{F}(p)$ denotes the family of all finite $p$-subgroups of $\Gamma$ and $n_{p}(\Gamma)$ the number of distinct conjugacy classes of elements of order a power of $p$ ( $p$ prime), then $R_{\mathscr{F}(p)}(\Gamma)$ can be defined and will be of rank $n_{p}(\Gamma)$. The following examples illustrate that these rings are readily computable from subgroup data, unlike the cohomology.

Example 1.4. $\Gamma=\mathrm{SL}_{2}(\mathbb{Z}), n(\Gamma)=8$, and

$$
R_{\mathscr{F}}\left(\mathrm{SL}_{2}(\mathbb{Z})\right) \cong \mathbb{Z}[w] / w^{8}+w^{6}-w^{2}-1=0 .
$$

Example 1.5. $\Gamma=\mathrm{SL}_{3}(\mathbb{Z}), n_{2}(\Gamma)=5$, and

$$
\begin{gathered}
\alpha_{1}^{2}=\alpha_{2}^{2}=1, \alpha_{1} \beta_{1}=\beta_{1} \\
\beta_{1}^{2}=2\left(1+\alpha_{1}\right), \beta_{2}^{2}=2\left(1+\alpha_{2}\right), \\
\alpha_{2} \beta_{2}=\beta_{2}, \alpha_{1} \alpha_{2}=\alpha_{1}+\alpha_{2}-1, \\
\alpha_{1} \beta_{2}=2 \alpha_{1}+\beta_{2}-2 \\
\beta_{1} \beta_{2}=2 \beta_{1}+2 \beta_{2}-4 \\
\alpha_{2} \beta_{1}=2 \alpha_{2}+\beta_{1}-2
\end{gathered}
$$

\section{Contribution to $K$-Theory}

For the sake of clarity of exposition, we work at a fixed prime $p$; let $K_{p}^{*}()$ denote $p$-adic $K$-theory and $\mathbb{C}_{p}$ the completion of the algebraic closure of $\mathbb{Q}_{p}$. We choose a fixed normal subgroup $\Gamma^{\prime} \subseteq \Gamma$ such that $\Gamma^{\prime}$ is torsion-free, so $G=\Gamma / \Gamma^{\prime}$ is finite. If $\gamma \in \Gamma$, let $C(\gamma)$ denote its centralizer; then it can be expressed as an extension

$$
1 \rightarrow C(\gamma) \cap \Gamma^{\prime} \rightarrow C(\gamma) \rightarrow H_{\gamma} \rightarrow 1
$$

where $\left|H_{\gamma}\right|<\infty$. Our main result is the following.

Theorem 2.1. Let $\Gamma$ be a discrete group of finite v.c.d. satisfying our finiteness assumptions. Then there is an exact sequence

$$
0 \rightarrow I_{p} \rightarrow K_{p}^{*}(B \Gamma) \otimes \mathbb{C}_{p} \stackrel{\varphi_{p}}{\longrightarrow} R_{\mathscr{F}(p)}(\Gamma) \otimes \mathbb{C}_{p} \rightarrow 0
$$

where $\varphi_{p}$ is a surjection of rings, and we have an additive decomposition

$$
I_{p} \cong \bigoplus_{(\gamma)} \tilde{K}_{p}^{*}\left(B\left(C(\gamma) \cap \Gamma^{\prime}\right)\right)^{H_{\gamma}} \otimes \mathbb{C}_{p}
$$

where the sum is taken over conjugacy classes of elements of order a finite power of $p$.

Corollary 2.2.

$$
K_{p}^{*}(B \Gamma) \otimes \mathbb{C}_{p} \cong R_{\mathscr{F}(p)}(\Gamma) \otimes \mathbb{C}_{p}
$$


if and only if $\tilde{H}^{*}(B C(\gamma), \mathbb{Q}) \equiv 0$ for every element $\gamma \in \Gamma$ of order a power of $p$.

The corollary follows from the fact that $I_{p}$ is determined by the cohomology of $B C(\gamma)$; it is, of course, independent of the choice of the extension.

Example 2.3. $\Gamma=G_{1} *_{H} G_{2}$ is an amalgamated product of finite groups. Then

$$
\begin{aligned}
& K_{p}^{0}(B \Gamma) \otimes \mathbb{C}_{p} \cong R_{\mathscr{F}(p)}(\Gamma) \otimes \mathbb{C}_{p}, \\
& K_{p}^{1}(B \Gamma) \otimes \mathbb{C}_{p} \cong\left(\mathbb{C}_{p}\right)^{v_{p}(\Gamma)}
\end{aligned}
$$

where

$$
v_{p}(\Gamma)=n_{p}(\Gamma)-n_{p}\left(G_{1}\right)-n_{p}\left(G_{2}\right)+n_{p}(H)
$$

represents the total sum of $\operatorname{dim}_{\mathbb{Q}} H^{1}(B C(\gamma), \mathbb{Q})$ as $\gamma \in \Gamma$ ranges over conjugacy classes of elements of order a power of $p$.

Example 2.4. $\Gamma=\mathrm{SL}_{3}(\mathbb{Z})$ and

$$
K_{2}^{*}(B \Gamma) \otimes \mathbb{C}_{2} \cong R_{\mathscr{F}(2)}(\Gamma) \otimes \mathbb{C}_{2},
$$

whence we can use Example 1.5 to determine this ring (compare with [So, TY]).

Example 2.5. $\Gamma=\mathrm{GL}_{p-1}(\mathbb{Z})$, where $p$ is odd prime. If $\mathrm{Cl}(p)=$ class number of $p$, then $R_{\mathscr{F}(p)}(\Gamma)$ can be computed from the extension

$$
0 \rightarrow R_{\mathscr{F}(p)}(\Gamma) \rightarrow\left(\bigoplus_{\mathrm{Cl}(p)} R(\mathbb{Z} / p)\right)^{\Delta} \rightarrow \mathbb{Z}^{t(p)-1} \rightarrow 0
$$

where $\Delta=$ Galois group and $t(p)=$ number of $\Delta$-orbits in the set of ideal classes. Hence $r k_{\mathbb{Z}} R_{\mathscr{F}(p)}(\Gamma)=1+\mathrm{Cl}(p)$, and in this case

$$
I_{p} \cong \tilde{K}_{p}^{*}\left(B \Gamma^{\prime}\right)^{G} \otimes \mathbb{C}_{p} \oplus\left(\bigoplus_{\mathrm{Cl}(p)} \tilde{K}_{p}^{*}\left(\left(S^{1}\right)^{(p-3) / 2}\right) \otimes \mathbb{C}_{p}\right)
$$

Sketch of Proof of 2.1. From a theorem of Serre [S] for the class of groups we consider that there exists a finite-dimensional $\Gamma$-complex $X$ with finite isotropy, contractible fixed point sets, and $X / \Gamma$ of finite type. By essentially identifying bundles that agree on finite subgroups, we construct a surjection of rings

$$
K_{\Gamma}^{*}(X) \rightarrow R_{\mathscr{F}}(\Gamma) .
$$

Next we identify $K_{\Gamma}^{*}(X) \cong K_{G}^{*}\left(X / \Gamma^{\prime}\right)\left(\Gamma^{\prime}, G\right.$ as before $)$ and use an additive decomposition for $K_{G}^{*}\left(X / \Gamma^{\prime}\right) \otimes \mathbb{C}$ obtained previously by the author [A] to estimate the kernel of this ring map in terms of the centralizers of elements of finite order in $\Gamma$.

The final technical step is to complete this map, as by the Atiyah-Segal completion theorem, $K_{G}^{*}\left(X / \Gamma^{\prime}\right)^{\wedge} \cong K^{*}(B \Gamma)$ (at $I G \subseteq R(G)$ ). Doing this locally leads to the statement in Theorem 2.1. 


\section{REFERENCES}

[A] A. Adem, On the K-theory of the classifying space of a discrete group, Math. Ann. 292 (1992), 319-327.

[At] M. F. Atiyah, Characters and cohomology of finite groups, Inst. Hautes Études Sci. Publ. Math., vol. 9, Presses Univ. France, Paris, 1961, pp. 23-64.

[B] K. Brown, High-dimensional cohomology of discrete groups, Proc. Nat. Acad. Sci. U.S.A. 73 (1976), 1795-97.

[F] F. T. Farrell, An extension of Tate cohomology to a class of infinite groups, J. Pure Appl. Algebra 10 (1977), 153-161.

[S] J.-P. Serre, Cohomologie des groupes discretes, Ann. of Math. Stud., vol. 70, Princeton Univ. Press, Princeton, NJ, 1971, pp. 77-169.

[So] C. Soulé, The cohomology of $\mathrm{SL}_{3}(\mathbb{Z})$, Topology 17 (1978), 1-22.

[TY] M. Tezuka and N. Yagita, Complex $K$-theory of $B \mathrm{BL}_{3}(\mathbb{Z})$, preprint 1992.

Department of Mathematics, University of Wisconsin, Madison, Wisconsin 53706

E-mail address: adem@math.wisc.edu 